\title{
Mortalidad materna Hospital Materno Infantil 1983-1984
}

\author{
Dres.: Guillermo Garcia Ojeda* , Heliodoro Ñañez V.**
}

\section{I - INTRODUCCION}

Este trabajo muestra los resultados obtenidos del estudio de los casos de mortalidad materna ocurridos en el Hospital Materno Infantil de Bogotá (antes denominado Instituto Materno Infantil I.M.I.), durante el período comprendido entre el 1o. de enero de 1983 y el 31 de diciembre de 1984.

En anteriores trabajos elaborados sobre este mismo tema, en las dos últimas décadas se han hecho análisis comparativos de las tasas de mortalidad materna con instituciones nacionales, del área latinoamericana y de hospitales de países de gran desarrollo económico y cultural, en donde se demuestra que la tasa de mortalidad materna y perinatal continúa siendo problema no resuel to de salud en nuestro medio, que reclama soluciones de todos y cada uno de los estamentos comprometidos en su atención, para me-

* Residente 3. Hospital Materno Infantil, Bogotá.

* Director del Departamento de Obstetricia y Ginecología de la Facultad de Medicina de la Universidad Nacional de Colombia. jorarla $(1,2,3,4,15,16,17,18,22$, 24).

Dentro dé la obstetricia actual, inan ido apareciendo nuevos enfoques de estudio y atención de la paciente embarazada, orientados de manera específica a identificar a la madre que corre un mayor riesgo de presentar patología o complicaciones durante su gestación, así como el de lograr que el fruto de dicho evento llegue a la vida en óptimas condiciones mentales y físicas.

Corresponde al obstetra y con él a las instituciones encargadas de prestar atención a la paciente grávida, procurar que estas situaciones se logren, lo cual no es fácil. Esto ha dado lugar a la aparición del concepto de "paciente de alto riesgo obstétrico" para identificarla y poder ofrecerle una atención selectiva en hospitales de tercer nivel y el surgimiento de la perinatología y la neonatología, como superespecialidades complementarias encamiriadas a una atención multidisciplinaria e integral de 1 : mujer gestante. Tal es el enfoque que se le ha dado al Hospital Materno Infantil de Bogotá y en el cual están comprometidos la Dirección del Hospital, el Departamento de Ginecoobstetricia, la Unidad de Recién nacidos y todos y cada uno de 
los elementos que lo integran. Si bien es cierto que con la remodelación física del Hospital, se dio un paso definitivo para lograr este objetivo fundamental desde el punto de vista locativo y de dotación, faltan aún elementos indispensables, como personal especializado en áreas paramédicas, dotación de elementos para el funcionamiento del Laboratorio Clínico, banco de sangre, unidad de cuidado intensivo y subsanar las fallas periódicas en el suministro de drogas $y$ de elementos de cirugía, que han hecho que la atención no haya podido darse en la forma prevista. Por otra parte, a pesar de que desde el punto de vista administrativo, existe una regionalización que daría a varias instituciones hospitalarias la responsabilidad de atención de tercer nivel, ésta en la práctica no se cumple $y$ ha hecho que sea el Hospital Materno Infantil quien deba asumirla. Estamos pues frente a un hospital de tercer nivel, con una infraestructura incompleta, que recibe el al to riesgo obstétrico de la población económicamente menos favorecida de Bogotá y que cuenta además con dos unidades únicas en su tipo y atención, la del Servicio de Sépticas y la del Servicio de Toxemias, a donde llegan pacientes extremadamente graves.

Lo anterior tiene como consecuencia que la Institución sea exclusivamente la que ésta soportando la atención de tercer nivel del área de Bogotá y Departamentos vecinos, ocasionando que los costos e insumos de atención sean tremendamente costosos $y$ que los índices de mortalidad materna y perinatal continúen siendo altos.

Con el presente estudio planeamos continuar con el análisis periódico de la mortalidad materna en nuestra Institución, con el fin de determinar la tasa de mortalidad materna, las causas determinantes de la misma, los principales factores de riesgo que la ocasionan y evaluar la calidad de atención Institucional y médica dada, con el fin de establecer estrategias normativas y preventivas.

\section{II - OBJETIVOS GENERALES}

1. Determinar la tasa de mortalidad materna en el Hospital Materno Infantil (HMI) durante los años 1983-1984.

2. Establecer las causas más frecuentes de mortalidad materna en el Hospital Materno Infantil (HMI) y analizar las variables determinantes.

3. Analizar la tasa y causas de muerte materna en el HMI durante los últimos 30 años.

4. Establecer una tasa de mortalidad materna depurada de la Institución.

\section{III - TIPO DE ESTUDIO - UNIVERSO}

El estudio es de tipo descriptivo retrospectivo. La población a estudiar está dada por las pacientes obstétricas fallecidas en el Hospital Materno Infantil (HMI) durante los años 1983-1984.

\section{IV - RECOLECCION DE LA INFORMACION - TABULACION}

La información requerida se obtuvo directamente de la Historia Clínica en el Departamento de Estadística y del archivo de Patología del Instituto. En la recolección de los datos se utilizó un formulario, del cual se adjunta copia, que contiene las variables establecidas en el protocolo para desarrollar el estudio. EI formulario consta de 3 partes a saber:

A. Identificación de la paciente.

B. Contenido de variables a estudiar.

C. Comentarios donde el encuestador hace las anotaciones o aclaraciones pertinentes. Las variables establecidas se 
han evaluado en estudios anteriores en el Hospital Materno Infantil (HMI).

Las causas de muerte están clasificadas según el listado aceptado por la Or. ganización Mundial de la Salud (OMS). Luego de diligenciar el formulario y de un juicio crítico de las variables se procesaron los datos, para obtener las frecuencias y las tablas necesarias para el análisis de la mortalidad materna en el período de estudio.

\section{V - DEFINICION DE CONCEPTOS Y VARIABLES}

\section{Muerte Materna}

“Es el fallecimiento de cualquier mujer por cualquier causa mientras está embarazada o dentro de cierto período después de la terminación del embarazo independiente de la duración o del sitio del mismo".

Este período fue aprobado por la FIGO de 42 días.

La muerte materna se clasifica en tres grupos:

\section{a. Muerte Materna directa:}

(Anteriormente mortalidad obstétrica)

"Es el fallecimiento resultante de complicaciones obstétricas del embarazo, trabajo de parto y puerperio; o intervenciones, omisiones de tratamiento necesario, tratamiento incorrecto o combinación de estas causas".

\section{b. Muerte Materna indirecta:}

(Anteriormente muerte obstétrica indirecta).

"Es el fallecimiento obstétrico resultante de enfermedad previa o enfermedad desarrollada durante el embarazo, parto o puerperio que no es debida directamente a causas obstétricas sino que es agravada por los efectos funcionales del embarazo".

\section{c. Muerte no Materna:}

(Anteriormente muerte no obstétrica).

"Fallecimiento obstétrico de causas accidentales o circunstanciales, no relacionadas con el embarazo o su tratamiento".

\section{Tasa de Mortalidad Materna:}

Es el número de muertes maternas directas, indirectas $\mathrm{y}$ no maternas por 10.000 nacidos vivos, en un período determinado.

\section{Nacidos Vivos:}

Se tuvo en cuenta la clasificación de la FIGO (1970).

"Es la expulsión o extracción completa del producto de la concepción que pese más de 1.000 gramos o tenga mas de 28 semanas de gestación; o en su defecto mida más de 35 centímetros $y$ que muestre señales de vida después de la separación de la madre".

\section{Edad:}

Años cumplidos de la paciente según registro de la Historia Clínica.

\section{Procedencia:}

Lugar en donde vivió la paciente en los últimos meses antes de su ingreso al Hospital.

\section{Remisión:}

Organismo de salud o persona que envió a la paciente al HMI. 


\section{Gestaciones:}

Número de embarazos anotados en la Historia Clínica.

\section{Edad Gestacional:}

Tiempo en semanas del embarazo al ingreso de la paciente en relación con la fecha de la última menstruación.

\section{Riesgo Obstétrico:}

Se refiere a la probabilidad de presentar complicaciones durante el embarazo, parto o puerperio.

\section{a. Bajo riesgo:}

Se clasifica así el embarazo en el cual la madre, el feto o el recién nacido tiene pocas posibilidades de presentar complicaciones.

\section{b. Alto riesgo:}

Se denomina embarazo de alto riesgo aquel en donde la madre, el feto o el recién nacido están o estarán expuestos a mayores peligros, en relación con un embarazo normal.

\section{Causas de muerte:}

Corresponde a la registrada en la hoja de egreso con base en el diagnóstico clínico y/o estudio de anatomía patológica.

\section{Muerte Institucional:}

Es la muerte materna ocurrida en el HMI después de 24 horas de hospitalización.

\section{Muerte no Institucional:}

Toda muerte materna ocurrida en el $\mathrm{HMI}$ antes de 24 horas de hospitalización, siempre y cuando la paciente haya ingresado con una complicación debida o no a su embarazo.

\section{Atención Médica:}

Se refiere a la calidad del manejo dispensado a cada paciente en cuanto al diagnóstico, tratamiento y/o seguimiento por parte del personal médico de la Institución. Tiene las siguientes subcategorías.

\section{a. Atención médica buena:}

Cuando el diagnóstico, tratamiento y/o seguimiento en cada paciente cumple con las normas establecidas en el Departamento de Ginecoobstetricia del HMI.

\section{b. Atención médica regular:}

Cuando existe error $u$ omisión en el diagnóstico, tratamiento y/o seguimiento de la paciente sin que ésto incidiera directamente en el resultado final.

\section{c. Atención médica mala:}

Siempre que se compruebe error $u$ omisión en el diagnóstico, tratamiento y/o seguimiento de la paciente $y$ esto influya en forma desfavorable en la evolución del estado clínico.

\section{Atención Institucional:}

Se refiere a la disponibilidad de los recursos humanos y físicos necesarios en el diagnóstico, tratamiento $\mathrm{y} / \mathrm{o}$ seguimiento de las pacientes que ingresan en el HMI.

Se consideran tres sub-categorías:

a. Atención institucional buena:

Es cuando el hospital cuenta con personal médico y paramédico suficiente: así como disponibilidad de elementos necesarios para un adecuado diagnóstico, 
tratamiento $y / o$ seguimiento de las pacientes que ingresan al servicio.

\section{b. Atencion institucional regular:}

Se clasifica así la atención institucional, cuando no existen todos los recursos humanos y físicos considerados necesarios en el manejo integral del paciente.

\section{c. Atención institucional mala:}

Cuando no se cuenta con los medios necesarios para el diagnóstico, tratamiento $y / o$ seguimiento de la paciente y esta situación incide negativamente en la evolución posterior.

\section{VI - PROCESAMIENTO Y ANALISIS DE LA INFORMACION}

Se revisaron tanto los archivos del Departamento de Estadística, como de Anatomía Patológica y se sometieron a estudio las muertes que ocurrieron en pacientes que estaban embarazadas o se hallaban en época de puerperio, en el lapso comprendido entre el 10. de enero de 1983 y el 31 de diciembre de 1984 . Para tal efecto se tuvieron en cuenta los resultados de autopsias, biopsias o piezas quirúrgicas $y$ en los casos en que estos datos no existían, se recurrio al diagnóstico clínico o quirúrgico que figura en la historia.

\section{VII - RESULTADOS}

\section{Tasa de mortalidad:}

En los dos (2) años estudiados (19831984), se registraron en el Instituto Materno Infantil, ochenta y siete muertes. de pacientes obstétricas (87). En estudios anteriores se excluyeron los casos que sobrepasaron los 42 días establecidos por la FIGC, pero en el presente tra- bajo ninguna paciente sobrepasó este límite, por lo cual no se da la discusión de si es lógico o no, tener en cuenta límites de tiempo para excluir estos casos de las investigaciones de mortalidad.

Durante el lapso del estudio ocurrieron veinte mil ochocientos noventa y un (20.891) nacimientos de niños vivos. De tal manera tenemos en el período revisado una tasa de mortalidad materna promediada de $41.64 \times 10.000$ nacidos vivos. Los datos anteriores se muestran por año en las tablas 1, 2 y 3 respectivamente.

Tabla No. 1

\begin{tabular}{lrrc}
\hline Año & 1983 & 1984 & $1983-1984$ \\
Muerte & 46 & 41 & 87 \\
\hline
\end{tabular}

Tabla No. 2

\begin{tabular}{lrrr}
\hline Año & 1983 & 1984 & $1983-1984$ \\
Nacidos vivos & 10.796 & 10.095 & 20.891 \\
\hline
\end{tabular}

Tabla No. 3

TASA POR AÑOS

1983

\begin{tabular}{l}
$\frac{\frac{46 \times 10.000}{10.796}=42.60 \times 10.000 \text { Nacidos vivos }}{1984}$ \\
$\frac{\frac{11 \times 10.000}{10.095}=40.61 \times 10.000 \text { Nacidos vivos }}{1983-1984}$ \\
$\frac{20.891}{87 \times 10.000}=41.64 \times 10.000$ Nacidos vivos \\
\hline
\end{tabular}




\section{Causas de mortalidad materna:}

Al agrupar las muertes de acuerdo a la clasificación de la FIGO, se encontró 69 de ellas de causa directa $(79.31 \%), 11$, de causa indirecta $(12.64 \%)$, y 7 de causa no materna (8.05\%). De uno a otro año hubo poca variación. Ver tabla 4.

Tabla No. 4

\section{CLASIFICACION DE LA MORTALIDAD}

\begin{tabular}{lrrrr}
\hline Año & 1983 & 1984 & TOTAL & PORCENTAJE \\
\hline Directa & 36 & 33 & 69 & 79.31 \\
Indirecta & 6 & 5 & 11 & 12.64 \\
No materna & 4 & 3 & 7 & 8.05 \\
\hline TOTAL & 46 & 41 & 87 & 100.00 \\
\hline
\end{tabular}

\section{a. Muerte materna directa:}

La mayor mortalidad se debió a complicaciones obstétricas ocurridas en los diferentes períodos de la gestación y el puerperio (69 casos).

Los factores desencadenantes del deceso se relacionan en la tabla 5 .

Tabla No. 5

\section{MUERTE MATERNA DIRECTA}

\begin{tabular}{lrrrc}
\hline Año & 1983 & 1984 & TOTAL & PORCENTAJE \\
\hline Infección & 22 & 21 & 43 & 62.31 \\
Toxemias & 6 & 7 & 13 & 18.84 \\
Hemorragia & 4 & 3 & 7 & 10.14 \\
Higado graso & 2 & 1 & 3 & 4.35 \\
Embolia amniótica & 2 & - & 2 & 2.89 \\
Enf. trofoblástica & - & 1 & 1 & 1.45 \\
\hline TOTAL & 36 & 33 & 69 & 100.00 \\
\hline
\end{tabular}

La infección como consecuencia del aborto, parto o cesárea ocupa el primer lugar de las causas directas (62.31\%), seguida de la toxemia (18.84\%), la hemorragia (10.14\%), el hígado graso
(4.35\%), la embolia del líquido amniótico $(2.89 \%)$ y la enfermedad trofoblástica $(1.45 \%)$.

\section{Mortalidad materna por infección:}

Del total de muertes maternas ocurridas en los dos años estudiados, 43 de ellas (49.42\%), tuvieron en la infección el origen de su defunción. La tabla 6 muestra el estado gestacional donde ésta se produjo.

Tabla No. 6

MORTALIDAD MATERNA POR INFECCION

\begin{tabular}{lrrrr}
\hline Año & 1983 & $\mathbf{1 9 8 4}$ & Total & Porcentaje \\
\hline Aborto & 15 & 18 & 33 & 76.74 \\
Puerperio & 3 & 2 & 5 & 11.63 \\
Cesárea & 3 & 1 & 4 & 9.31 \\
Embarazo ectópico & 1 & - & 1 & 2.32 \\
\hline TOTAL & 22 & 21 & 43 & 100.00 \\
\hline
\end{tabular}

De las 33 pacientes que abortaron, refirieron maniobras abortivas 28 ( $84 \%$ ) y se encontró perforación del útero en ? (21\%). La mayoría consultaron por aborto incompleto (93\%).

De las 5 puerperas que murieron por infección, 4 ( $80 \%$ ) habian tenido el parto fuera del hospital e ingresaron infectadas a éste.

De las 4 pacientes infectadas en el postoperatorio de cesárea y que murieron, a 2 (50\%) se les había practicado la primera cesárea y a las otras 2 (50 \%) cesárea iterativa; las 4 tuvieron dehiscencia de la histerorrafia.

En 1984 se observó un ligero incremento del aborto infectado con relación a 1983 y una disminución de la infección en el puerperio $y$ en el postoperatorio de cesárea, relacionado posiblemente esto último con la remodelación del hospi- 
tal, que disminuyó el hacinamiento de pacientes.

En el Servicio de Sépticas del Hospital Materno Infantil se utiliza la siguiente clasificación de la infección: (12).

Estado I: Infección localizada a nivel del útero

la: El foco séptico se encuentra en el endometrio

Ib: Miometritis Séptica aguda

Estado II: Infección localizada en anexos, parametrios y paracolpos.

Ila: Endosalpingitis aguda

Ilb: Presencia de empastamientos o masas anexiales

IIlc: Celulitis pélvica aguda

Estado III: Infección localizada en la serosa peritoneal

Illa: Pelviperitonitis

IIlb: Peritonitis generalizada

Estado IV: Sepsis generalizada

IVa: Septicemia

IVb: Shock séptico.

Teniendo en cuenta que en la mayoría de casos, coinciden dos o más estados sépticos, que sumados a las complicaciones de la infección, !levan a la muerte de las pacientes, se investigó la presencia de cada uno de estos estados, independientemente de que coincidieran uno o más de ellos. Los datos se muestran en la tabla 7.

Los estados sépticos más frecuentes fueron: la miometritis ( $55.81 \%$ ), la peri- tonitis (53.48\%) y el shock séptico (51.16\%); seguidos por la septicemia $(34.88 \%)$, la endometritis $(27.90 \%)$, el absceso tuvo-ovárico ( $1 \mathrm{lb}$ ) y la salpingitis. Los 3 primeros estados explican la alta frecuencia de muerte de estas pacientes.

Tabla No. 7

ESTADOS SEPTICOS

\begin{tabular}{lrrrr}
\hline Afio & $\mathbf{1 9 8 3}$ & $\mathbf{1 9 8 4}$ & Total & Porcentaje \\
\hline Miometritis & 9 & 15 & 24 & 55.81 \\
Peritonitis & 14 & 9 & 23 & 53.48 \\
Shock sẻptico & 10 & 12 & 22 & 51.16 \\
Septicemia & 7 & 8 & 15 & 34.88 \\
Endometritis & 6 & 6 & 12 & 27.90 \\
Absceso tubo-ovárico & 8 & 3 & 11 & 25.58 \\
Salpingitis & 2 & 4 & 6 & 13.95 \\
\hline
\end{tabular}

Las complicaciones derivadas de la infección se presentan en la tabla 8 .

Tabia No. 8

COMPLICACIONES DE LA INFECCION

\begin{tabular}{lcccc}
\hline Año & 1983 & 1984 & Total & Porcentaje \\
\hline $\begin{array}{l}\text { Síndrome dificultad respiratoria } \\
\text { del adulto }\end{array}$ & 9 & 5 & 14 & 32.55 \\
Insuficiencia renal aguda & 9 & 5 & 14 & 32.55 \\
Tromboembolismo pulmonar & 5 & 7 & 12 & 27.90 \\
Trombosis infundíbulo pélvico & 2 & 8 & 10 & 23.25 \\
Edema pulmonar & 6 & 2 & 8 & 18.60 \\
$\begin{array}{l}\text { Coagulación intravascular } \\
\text { diseminada }\end{array}$ & 4 & 3 & 7 & 16.27 \\
Fistulas enterocu táneas & 1 & 1 & 2 & 4.65 \\
Insuficiencia cardíaca & - & 2 & 2 & 4.65 \\
Edema cerebral & 1 & - & 1 & 2.32 \\
\hline
\end{tabular}

De los datos anteriores, se destaca que por lo menos las 5 complicaciones más frecuentes requieren para su manejo adecuado una Unidad de Cuidado Intensivo de la cual actualmente no dispone la Institución. 


\section{Mortalidad Materna por Toxemia:}

La toxemia ocupó el segundo lugar como causa de muerte materna en general $(14.94 \%)$ y como causa de muerte materna directa $(18.84 \%)$. Se excluyeron los casos con antecedentes de enfermedad vascular hipertensiva crónica (EVHC) y sólo se tuvieron en cuenta los de toxemia pura.

Se presentó como causa de muerte materna en 13 casos, fue más frecuente entre las 37 y 40 semanas de gestación (61.53\%). Se encontró como eclampsia en 12 casos (92.31\%), principalmente en el preparto (92.31\%). Ver tabla 9.

Tabla No. 9

MORTALIDAD MATERNA POR TOXEMIA

\begin{tabular}{lrrrr}
\hline Año & $\mathbf{1 9 8 3}$ & $\mathbf{1 9 8 4}$ & Total & Porcentaje \\
\hline Tipo Preeclamṕsia & 1 & - & 1 & 7.69 \\
Eclampsia & 5 & 7 & 12 & 92.31 \\
Aparición Preparto & 5 & 7 & 12 & 92.31 \\
Intraparto & 1 & - & 1 & 7.69 \\
\hline \multicolumn{1}{c}{ Total } & 6 & 7 & 13 & 100.00 \\
\hline
\end{tabular}

La toxemia se complicó con insuficiencia renal aguda en 10 casos (76.92잉, edema pulmonar en 9 (69.23\%), accidente cerebro-vascular en $9(69.23 \%)$, edema cerebral en 7 (53.85\%), hemorragia subcapsular hepática en 6 (46.15\%), coagulación intravascular diseminada en 5 (38.46\%) y abruptio placentae en $1 \mathrm{ca}$ so $(7.69 \%)$. Ver tabla 10.

\section{Mortalidad Materna por Hemorragia:}

La hemorragia como causa de muerte ocupó el tercer lugar de las causas directas $(10.14 \%)$ y también el tercer lugar dentro de la mortalidad global (8.04\%). En la tabla 11 se señalan las cau- sas de los 7 casos de hemorragia que se presentaron en los 2 años.

Tabla No. 10

COMPLICACIONES DE LA TOXEMIA

\begin{tabular}{lcrrr}
\hline Año & 1983 & 1984 & Total & Porcentaje \\
\hline $\begin{array}{l}\text { Insuficiencia renal aguda } \\
\text { Edema pulmonar }\end{array}$ & 4 & 6 & 10 & 76.92 \\
& 4 & 5 & 9 & 69.23 \\
$\begin{array}{l}\text { Accidente cerebrovascular } \\
\text { Edema cerebral }\end{array}$ & 3 & 6 & 9 & 69.23 \\
$\begin{array}{l}\text { Hemorragia subcapsular } \\
\text { hepática }\end{array}$ & 2 & 5 & 7 & 53.85 \\
$\begin{array}{l}\text { Coagulación intravascular } \\
\text { diseminada }\end{array}$ & 4 & 2 & 6 & 46.15 \\
Abruptio placentae & - & 5 & 5 & 38.46 \\
\hline Total & - & 1 & 1 & 7.69 \\
\hline
\end{tabular}

\section{b. Muerte materna indirecta:}

Se estableció la existencia de una patología previa al embarazo, agravada por el estado grávido-puerperal en 11 casos $(12.64 \%)$, los cuales se relacionan en la tabla 12 .

La enfermedad vascular hipertensiva crónica (EVHC) encabezó con 4 casos $(36.36 \%)$, las causas de muerte indirecta $y$ se debe tener en cuenta que el embarazo agrava esta entidad, por lo cual las nuertes eran evitables si no hubiera ocuirido la aestación.

Tabla No. 11

\section{MORTALIDAD MATERNA POR HEMORRAGIA}

\begin{tabular}{lcccc}
\hline Año & 1983 & 1984 & Total & Porcentaje \\
\hline Desgarro cesárea & 2 & - & 2 & 28.57 \\
Atonía uterina & - & 1 & 1 & 14.28 \\
Acretismo placent. & 1 & - & 1 & 14.28 \\
Alumbramiento incomple to & 1 & - & 1 & 14.28 \\
Abruptio placentae & - & 1 & 1 & 14.28 \\
Embarazo Ectópico & - & 1 & 1 & 14.28 \\
\hline Total & 4 & 3 & 7 & 100.00 \\
\hline
\end{tabular}


Tabla No. 12

\section{MUERTE MATERNA INDIRECTA}

\begin{tabular}{lcccr}
\hline Año & $\mathbf{1 9 8 3}$ & $\mathbf{1 9 8 4}$ & Total & Porcentaja \\
\hline Enfermedad vascular hiperten- & & & & \\
siva crónica & 2 & 2 & 4 & 36.36 \\
Enfermedad cardiorespiratoria & 1 & - & 1 & 9.09 \\
Tuberculosis & 1 & - & 1 & 9.09 \\
Pielonefritis & - & 1 & 1 & 9.09 \\
Glomerulonefritis & - & 1 & 1 & 9.09 \\
Síndrome varicoso severo & 1 & - & 1 & 9.09 \\
Leucemia & 1 & - & 1 & 9.09 \\
Valvulopat ia aórtica & - & 1 & 1 & 9.09 \\
\hline Total & 6 & 5 & 11 & 100.00 \\
\hline
\end{tabular}

\section{c. Muerte no Materna:}

Hubo 7 casos de muertes no relacionadas con el embarazo (8.05\%) y que se presentan en la tabla 13.

Tabla No. 13

MUERTE NO MATERNA

\begin{tabular}{lcccr}
\hline Año & 1983 & 1984 & Total & Porcentaje \\
\hline Accidente anestésico & 1 & 1 & 2 & 28.57 \\
Status epiléptico & 1 & - & 1 & 14.28 \\
Cáncer de cérvix & 1 & - & 1 & 14.28 \\
Ruptura aneurisma & 1 & - & 1 & 14.28 \\
Meningitis & - & 1 & 1 & 14.28 \\
Osteosarcoma & - & 1 & 1 & 14.28 \\
\hline Total & 4 & 3 & 7 & 100.00 \\
\hline
\end{tabular}

El accidente anestésico, se tomó como causa de muerte no materna y se presentó en 2 casos $(28.57 \%)$.

\section{Mortalidad materna por Anestesia:}

Son varios los eventos que ocurren durante una anestesia: accidente anestésico como causa de muerte no materna, accidente anestésico no mortal, paro cardíaco intraoperatorio y muerte intraoperatoria, independientes de la calidad de la anestesia. Cada uno de estos eventos se analizó en los casos de las pacientes fallecidas, incluidas en el estudio y se presentan en la tabla 14.
Tabla No. 14

\section{EVENTOS ASOCIADOS A LA ANESTESIA}

\begin{tabular}{lrrrr}
\hline Año & 1983 & 1984 & Total & Porcentaje \\
\hline Paro cardíaco & 4 & 5 & 9 & 40.90 \\
Muerte intraoperatoria & 3 & 6 & 9 & 40.90 \\
Accidente anestésico mortal & 1 & 1 & 2 & 9.10 \\
Accidente anestésico no mortal & 1 & 1 & 2 & 9.10 \\
\hline Total & 9 & 13 & 22 & 100.00 \\
\hline
\end{tabular}

En 22 pacientes se encontraron eventos asociados a la anestesia (25.28\%). Se presentó paro cardíaco durante la anestesia en 9 casos (40.90\%), pero no se tuvieron en cuenta como accidente anestésico, ya que las pacientes sufrían entidades previas, que facilitaron el paro cardíaco, que se presentó por éstas sin que pudiera afirmarse que el procedimiento anestésico, hubiera tenido efecto directo sobre su ocurrencia.

Fallecieron durante el acto anestésico-operatorio 9 pacientes $(40.90 \%)$, pero se pensó que la muerte se presentó como efecto del pésimo estado general de las enfermas y no de la anestesia en sí misma. El accidente anestésico ocurrió en 4 casos (18.20\%), 2 de los cuales fueron extra-hospitalarios (9.10\%) no mortales, llegaron remitidos al $\mathrm{HMI}$ y posteriormente fallecieron por otra causa. En la tabla 15 , se indican la etapa y la causa de estos accidentes anestésicos. Se llegó a esta conclusión, luego de amplia discusión conjunta en los Departamentos de Obstetricia y Anestesia, de cada caso en particular.

Tabla No. 15

\section{ACCIDENTE ANESTESICO}

\begin{tabular}{lrrrr} 
Año & 1983 & $\mathbf{1 9 8 4}$ & Total & Porcentaje \\
\hline Intrahospitalario mortal & 1 & 1 & 2 & 50.00 \\
$\begin{array}{l}\text { Extrahospitalario no mortal } \\
\text { Intraoperatorio }\end{array}$ & 1 & 1 & 2 & 50.00 \\
$\begin{array}{l}\text { Por falla en la valoración pre- } \\
\text { anestésica }\end{array}$ & 2 & 2 & 4 & 100.00 \\
$\begin{array}{l}\text { Por error en la elección de la } \\
\text { anestesia }\end{array}$ & 1 & 2 & 3 & 75.00 \\
\hline
\end{tabular}


En el estudio no se incluyen casos de accidente anestésico no mortal intrahospitalario y en los cuales la paciente no falleció después, por lo que es de suponer una alta frecuencia de este tipo de accidentes que al parecer no se están cuantificando adecuadamente en la Institución.

\section{Mortaliciad materna en relación con:}

\section{a. Procedencia:}

En la tabla 16 se señala como del total de 87 pacientes que murieron en el HMI durante el período estudiado, el $79.32 \%$ (69 pacientes) procedían de la ciudad de Bogotá, un 18.39\% (16 pacientes) venían remitidas de Cundinamarca y otros Departamentos, y el un 2.29\%, ( 2 pacientes) no se estableció el lugar de orígen de la paciente.

Tabla No. 16

\section{PROCEDENCIA}

\begin{tabular}{lrrrr}
\hline Año & 1983 & 1984 & Total & Porcentaje \\
\hline Bogotá & 34 & 35 & 69 & 79.32 \\
Cundinamarca & 8 & 3 & 11 & 12.65 \\
Meta & - & 2 & 2 & 2.29 \\
Casanare & - & 1 & 1 & 1.15 \\
Boyacá & 1 & - & 1 & 1.15 \\
Caque tá & 1 & - & 1 & 1.15 \\
Sin dato & 2 & - & 2 & 2.29 \\
\hline Total & 46 & 41 & 87 & 100.00 \\
\hline
\end{tabular}

En estos datos se aprecia que el Hospital Materno Infantil, además de presentar un cubrimiento asistencial a la ciudad de Bogotá, brinda atención a pacientes de alto riesgo de sitios distantes como Caquetá y Casanare, demostrando la falta de recursos para la atención de estas pacientes en su lugar de origen, el aumento de los costos que representa su traslado y el centralismo en la atención médica.

\section{b. Lugar de Remisión:}

En la tabla 17, se observa como de las 87 pacientes fallecidas en los 2 años, solamente 6 (6.89\%) habian consultado previamente en los servicios del complejo hospitalario San Juan de Dios - Hospital Materno Infantil. En 22 pacientes (25.28\%) no se conoció dato de remisión y por lo tanto se consideraron como pacientes nuevas en la Institución.

Tabla No. 17

LUGAR DE REMISICN

\begin{tabular}{lrrrr}
\hline Año & 1983 & 1984 & Total & Porcentaje \\
\hline HMI & 3 & 3 & 6 & 6.89 \\
Hospital regional & 13 & 10 & 23 & 26.44 \\
Centro de salud & 8 & 9 & 17 & 19.55 \\
Hospital local & 2 & 6 & 8 & 9.19 \\
Médico particular & - & 1 & 1 & 1.15 \\
Hospital universitario & 4 & 2 & 6 & 6.90 \\
Clínica privada & 3 & 1 & 4 & 4.60 \\
Sin dato & 13 & 9 & 22 & 25.28 \\
\hline Total & 46 & 41 & 87 & 100.00 \\
\hline
\end{tabular}

59 pacientes $(67.82 \%)$ venian remitidos de otras Instituciones, entre las cuales se encontraron distintos niveles del Sistema Nacional de Salud en 54 casos (62.08\%) y aún servicios privados en 5 casos $(5.75 \%)$.

Se resal ta que una cuarta parte de las pacientes $(26.44 \%$ ) procedían de hospitales regionales y 6 pacientes $(6.90 \%$ ) venían de hospitales universitarios, sin contar las de su área de influencia. Es decir, el nivel más al to del Sistema Nacional de Salud, donde existe la infraestructura adecuada para la atención de estos casos, no justificàndose el que estas pacientes recurran al Hospital Materno Infantil, existiendo estas instituciones. Esto conlleva a un incremento en las tasas de mortalidad del HMI y a un descenso ficticio de las cifras de mortalidad de otras instituciones; dando la falsa imnre- 
sión de mala atención en el HMI y de buena atención en las otras entidades, cuando lo que realmente ocurre es la negación de un servicio médico asistencial, que es posible en éstas últimas.

\section{c. Edad y causa de muerte:}

La mortalidad materna según edad y causa se relaciona en la tabla 18. En los dos años del estudio la frecuencia máxima de muertes, se observó en mujeres entre los 20 y 24 años (28.73\%), seguida por el grupo de 25 a 29 años (22.99\%) $y$ el de 30 a 34 años (21.85\%). En estos tres grupos la causa de muerte fue direc- ta a expensas de la infección, seguida por la toxemia, la enfermedad vascular hipertensiva crónica y la hemorragia.

\section{d. Número de gestaciones y causa de muerte:}

La mayoría de defunciones maternas en el $\mathrm{HMI}$ se presentaron en primegestantes $(24.14 \%$ ) y se observó una disminución progresiva a medida que aumentaba la paridad.

La mayor paridad se presentó en una paciente con 16 gestaciones. En 2 casos no se logró establecer la paridad.

Tabla No. 18

EDAD Y CAUSA DE MUERTE

\begin{tabular}{lcccccccc}
\hline Edad & Infección & Toxemia & Hemorragia & Otras Indirecta & No obstétrica & Total & Porcentaje \\
\hline $15-19$ & 5 & 2 & - & 1 & - & 1 & 9 & 10.34 \\
$20-24$ & 13 & 3 & 3 & 2 & 3 & 1 & 25 & 28.73 \\
$25-29$ & 12 & 3 & 2 & - & 3 & - & 20 & 22.99 \\
$30-34$ & 8 & 4 & 1 & 2 & 2 & 2 & 19 & 21.85 \\
23 y más & 5 & 1 & 1 & 1 & 3 & 3 & 14 & 16.09 \\
\hline Total & 43 & 13 & 7 & 6 & 11 & 7 & 87 & 100.00 \\
\hline
\end{tabular}

Tabla No. 19

NUMERO DE GESTACIONES Y CAUSA DE MUERTE

\begin{tabular}{crrrrrrrrr}
\hline Gestaciones & Infección & Toxemia & Hemorragia & Otras & Indirecta & No obstétrica & Total & Porcentaje \\
\hline G1 & 6 & 8 & 1 & 3 & 1 & 2 & 21 & 24.14 \\
G2 & 6 & 3 & 3 & 1 & 3 & 2 & 18 & 20.69 \\
G3 & 14 & 1 & 1 & 1 & 1 & 1 & 19 & 21.84 \\
G4 & 4 & - & - & - & - & 1 & 5 & 5.75 \\
G5 y más & 12 & 1 & 1 & 1 & 6 & 1 & 22 & 25.29 \\
Sin dato & 1 & - & 1 & - & - & - & 2 & 2.29 \\
\hline Total & 43 & 13 & 7 & 6 & 11 & 7 & 87 & 100.00 \\
\hline
\end{tabular}


La mortalidad materna según el número de gestaciones y la causa de muerte se relaciona en la tabla 19.

Si se reduce a tres los grupos de paridad de las pacientes, se encuentra que 42 pacientes $(48.28 \%)$ tenían entre 2 y 4 embarazos, 22 pacientes $(25.29 \%)$ eran grandes multíparas (5 o más embarazos), y 21 eran primigestantes, (24.14ㅇ).

Al sumar las cifras de todas las multíparas, se encontró que un $73.57 \%$ (64 pacientes) dejaron con su fallecimiento un gran impacto social, ya que al tener en cuenta también la edad $(62.06 \%$ entre 15 y 30 años) se puede deducir la gran cantidad de hijos de corta edad que quedan huérfanos.

\section{e. Periodo gestacional y causa de muerte:}

Se analiza en la tabla 20.

El $40.23 \%$ de las muertes maternas ocurrió cuando la paciente cursaba el primer trimestre de embarazo; a consecuencia del aborto incompleto infectado en 33 casos, hemorragia por embarazo ectópico en 1 caso y por status epiléptico en otro caso.

En el 25.29\% la complicación determinante de la muerte se presentó en el puerperio. Predominó en este período la infección ( 7 casos), seguida por la enfermedad vascular hipertensiva crónica (5 casos), la toxemia (4 casos) y la hemorragia (4 casos).

En el tercer trimestre murieron igual número de pacientes que en el puerperio (25.29\%). En esta etapa del embarazo las muertes se asociaron principalmente a complicaciones de la toxemia (9 casos) $y$ de la enfermedad vascular hipertensiva crónica (4 casos).

\section{f. Datos al ingreso:}

Se señalan en la tabla 21.

Tabla No. 21

DATOS AL INGRESO

\begin{tabular}{llrrlr}
\hline Año & & $\mathbf{1 9 8 3}$ & $\mathbf{1 9 8 4}$ & Total & Porcentaje \\
\hline Prenatal & $\mathrm{Si}$ & 9 & 7 & 16 & 18.40 \\
& No & 37 & 34 & 71 & 81.60 \\
Temperatura: & Alta & 13 & 12 & 25 & 28.74 \\
& Baja & 8 & 5 & 13 & 14.94 \\
& Normal & 25 & 24 & 49 & 56.32 \\
Consciente & Si & 35 & 31 & 66 & 75.86 \\
& No & 11 & 10 & 21 & 24.13 \\
Tensión & Alta & 11 & 11 & 22 & 25.28 \\
Arterial & Baja & 15 & 13 & 28 & 32.19 \\
& Normal & 20 & 17 & 37 & 42.53 \\
\hline
\end{tabular}

Dado el alto número de pacientes remitidas que llegan al HMI, es importante

Tabla No. 20

PERIODO GESTACIONAL Y CAUSA DE MUERTE

\begin{tabular}{lccccccrr}
\hline Estadio & Infección & Toxemia & Hemorragia & Otras & Indirecta & No obstétrica & Total & Porcentaje \\
\hline 1o. trim. & 33 & - & 1 & - & - & 1 & 35 & 40.23 \\
2o. trim. & 2 & - & - & 2 & 2 & 2 & 8 & 9.19 \\
3o. trim. & 1 & 9 & 2 & 3 & 4 & 3 & 22 & 25.29 \\
Puerperio & 7 & 4 & 4 & 1 & 5 & 1 & 22 & 25.29 \\
\hline Total & 43 & 13 & 7 & 6 & 11 & 7 & 87 & 100.00 \\
\hline
\end{tabular}


establecer algunos datos del ingreso de las pacientes, por lo cual se analizaron variables tales como el antecedente de prenatal y signos vitales básicos como temperatura, estado de conciencia y cifras tensionales en el momento de la admisión al hospital. Esto nos permite tomar una idea del deterioro del estado de salud de las pacientes cuando consultan.

En el período 1983-1984, el $81.50 \%$ (71 pacientes) no tuvieron ningún tipo de control médico durante el tiempo de gestación previo a la hospitalización.

La temperatura se encontró alta en el $28.74 \%$ ( 25 pacientes), baja en el $14.94 \%$ (13 pacientes) y normal en el $56.32 \%$ (49 pacientes).

El estado de conciencia se encontró al terado al ingreso en el 24.13\% (21 pacientes).

La tensión arterial se encontró alta en la primera consulta en el 25.28\% (22 pacientes), baja en el $32.19 \%$ (28 pacientes) y normal en el $42.53 \%$ (37 pacientes).

\section{g. Parto:}

Los datos relacionados con el parto se señalan en la tabla 22.

Tabla No. 22

\section{PARTO}

\begin{tabular}{lrrrr}
\hline Año & 1983 & 1984 & Total & Porcentaje \\
\hline Espontáneo & 12 & 8 & 20 & 37.73 \\
Fuera del hospital & 11 & 5 & 16 & 30.18 \\
Cesárea & 8 & 4 & 12 & 22.64 \\
Refuerzo ocitócico & 1 & 3 & 4 & 7.54 \\
Espátulas & 2 & 1 & 3 & 5.66 \\
Fórceps & - & 2 & 2 & 3.77 \\
Gemelar & 1 & - & 1 & 1.88 \\
Total de partos & 33 & 20 & 53 & 60.91 \\
Total de muertes & 46 & 41 & 87 & 100.00 \\
\hline
\end{tabular}

Durante el período en estudio, tuvieron el parto 53 pacientes $(60.91 \%)$, de las 87 fallecidas, de las cuales 16 $(30.18 \%$ ) fueron atendidas fuera del Hospital Materno Infantil y las 37 restantes (69.82\%) en la Institución. De estas últimas tuvieron parto espontáneo 20 (37.73\%) e intervenido 17 (32.07\%). A $12(22.64 \%$ ) se les practicó cesárea, a 3 (5.66\%) aplicación de espátulas de Velasco y a 2 (3.77\%) fórceps. En 4 pacientes (7.54\%) se utilizó refuerzo del trabajo con ocitócico y se presentó un caso de embarazo gemelar (1.88\%).

\section{h. Complicaciones del parto:}

Las complicaciones del parto se indican en la tabla 23.

Tabla No. 23

\section{COMPLICACIONES DEL PARTO}

\begin{tabular}{lrrrr}
\hline Año & $\mathbf{1 9 8 3}$ & $\mathbf{1 9 8 4}$ & Total & Porcentaje \\
\hline Muerte perinatal & 10 & 8 & 18 & 33.96 \\
Retención de restos & 8 & 3 & 11 & 20.75 \\
RPM & 5 & 4 & 9 & 16.98 \\
Hipovolemia & 6 & - & 6 & 11.32 \\
Presentación distócica & 2 & 2 & 4 & 7.54 \\
Desgarros & 1 & 1 & 2 & 3.77 \\
Acretismo placentario & 1 & - & 1 & 1.88 \\
Ruptura uterina & - & 1 & 1 & 1.88 \\
Total partos & 33 & 20 & 53 & 100.00 \\
Partos complicados & 22 & 15 & 37 & 69.81 \\
Total muertes & 46 & 41 & 87 & 100.00 \\
\hline
\end{tabular}

De las 53 pacientes que tuvieron el parto, éste presentó 1 o más complicaciones en 37 casos (69.81\%), tales como retención de restos placentarios en 11 casos (20.75\%), ruptura prematura de membrana (RPM) en 9 (16.98\%), hipovolemia en 6 (11.32\%), presentación distócica en $4(7.54 \%)$, desgarro del canal del parto en 2 (3.77\%), acretismo placentario en 1 (1.88\%) y ruptura uterina en 1 caso ( $1.88 \%$ ). 
De los 54 productos de la gestación, se presentó mortalidad perinatal en la tercera parte de ellos (18).

\section{i. Anestesia:}

De las 87 pacientes fallecidas en los 2 años, a 60 (68.96\%) se les aplicó anestesia. Una sola vez a $24(27.58 \%)$, 2 veces a $23(26.43 \%), 3$ veces a $8(9.19 \%)$, 4 veces a $4(4.59 \%)$ y 5 veces en 1 caso (1.14\%). La anestesia más usada fue la general (101), luego la peridural (8), la raquidea (3) y combinada (1). En total se aplicaron 113 anestesias. Los procedimientos más frecuentes practicados durante las anestesias fueron: laparotomías 60 (53.09\%), legrados 21 (18.59\%), revisiones uterinas $17(15.03 \%)$ y cesáreas $12(10.62 \%)$.

Tabla No. 24

\section{ANESTESIA}

\begin{tabular}{llrrrr}
\hline & Año & 1983 & 1984 & Total & Porcontaje \\
\hline \multirow{6}{*}{ Número } & Si recibieron & 32 & 28 & 60 & 68.96 \\
& No recibieron & 14 & 13 & 27 & 31.04 \\
& 1 vez & 13 & 11 & 24 & 27.58 \\
de & 2 veces & 14 & 9 & 23 & 26.43 \\
anestesias & 3 veces & 3 & 5 & 8 & 9.19 \\
& 4 veces & 2 & 2 & 4 & 4.59 \\
& 5 veces & - & 1 & 1 & 1.14 \\
Tipo de & General & 48 & 53 & 101 & 89.38 \\
anestesia & Peridural & 7 & 1 & 8 & 7.07 \\
& Raquidea & 1 & 2 & 3 & 2.65 \\
& Combinada & 1 & - & 1 & 0.88 \\
\hline \multirow{4}{*}{} & Total & 57 & 56 & 113 & 100.00 \\
\hline
\end{tabular}

Los datos relacionados con las anestesias y sus indicaciones se muestran en las tablas 24 y 25.

\section{j. Riesgo obstétrico:}

De acuerdo con los parámetros de clasificación del riesgo obstétrico existentes en el hospital, !as pacientes se clasifican al ingreso como de alto, medio y bajo riesgo. En el presente estudio todas las pacientes fueron de alto riesgo.
Tabla No. 25

\section{INDICACION DE LA ANESTESIA}

\begin{tabular}{lrrrr}
\hline Año & 1983 & 1984 & Total & Porcentaje \\
\hline Laparatom la & 30 & 30 & 60 & 53.09 \\
Legrado & 9 & 12 & 21 & 18.59 \\
Revisión uterina & 8 & 9 & 17 & 15.03 \\
Cesárea & 8 & 4 & 12 & 10.62 \\
Trabajo de parto & 1 & - & 1 & 0.88 \\
Espátulas & - & 1 & 1 & 0.88 \\
Examen bajo anestesia & 1 & - & 1 & 0.88 \\
\hline Total anestesias & 57 & 56 & 113 & 100.00 \\
\hline
\end{tabular}

\section{Anatomía Patológica:}

El examen de anatomía patológica se hizo a partir de especímenes obtenidos después de intervenciones quirúrgicas y/o práctica de autopsia en algunas de las pacientes muertas.

En la tabla 26 se relacionan los estudios practicados.

Tabla No. 26

ANATOMIA PATOLOGICA

\begin{tabular}{lrr}
\hline & Casos & Porcentaje \\
\hline Autopsia & 47 & 54.02 \\
Pieza quirúrgica solamente & 8 & 9.20 \\
Pieza quirúrgica más autopsia & 8 & 9.20 \\
Sin estudio & 24 & 27.58 \\
\hline
\end{tabular}

Los resultados obtenidos muestran como en el $72.42 \%$ de las pacientes fallecidas fue posible confirmar el diagnóstico clínico, con base en uno o dos estudios histopatológicos.

En 24 casos (27.58\%) no se practicó autopsia; de ellos 16 por levantamiento médico-legal, 3 por oposición familiar y en 5 no se pudo establecer por qué no se hizo el estudio anatomopatológico.

\section{Diagnóstico:}

Confrontando el diagnóstico con los hallazgos quirúrgicos, de laboratorio $y$ 
de anatomía patológica, se evaluó el grado de certeza del diagnóstico clínico.

En los casos en que no coincidió con esta confirmación, se consideró errado el diagnóstico y como hay dos momentos en que esto es más importante, para la terapia de las pacientes, se calificó este error al inicio $\mathrm{y} / \mathrm{o}$ al final. Al principio porque es el momento en que se traza la línea de manejo del caso y al final porque es el momento en que la paciente fallece cuando se agotan todos los recursos de que dispone el médico y la Institución para tratar de recuperar a la paciente. En la tabla 27 se señalan los resultados de éste análisis.

Tabla No. 27

DIAGNOSTICO

\begin{tabular}{lrrrr}
\hline Año & 1983 & 1984 & Total & Porcentaje \\
\hline Acertado & 34 & 33 & 67 & 77.02 \\
Errado al inicio & 7 & 6 & 13 & 14.94 \\
Errado al final & 5 & 2 & 7 & 8.04 \\
\hline Total & 46 & 41 & 87 & 100.00 \\
\hline
\end{tabular}

Hubo error de diagnóstico en 20 casos (22.98\%): al principio en 13 (14.94\%) $y$ al final en 7 (8.04\%).

Se acertó en el diagnóstico en 67 pacientes $(77.02 \%)$ y esto llevó a un manejo ajustado a las necesidades de las enfermas en la mayoría de los casos.

\section{Historia clínica:}

La calidad de la historia clínica se señala en la tabla 28.

En la revisión se encontró que la calidad de la historia fue buena en 68 casos (78.16\%), regular en $16(18.39 \%)$ y mala en 3 casos (3.45\%).

\section{Atención médica:}

Se analiza en la tabla 29.
Tabla No. 28

HISTORIA CLINICA

\begin{tabular}{lcrrr}
\hline Año & 1983 & 1984 & Total & Porcentaje \\
\hline Buena & 33 & 35 & 68 & 78.16 \\
Regular & 12 & 4 & 16 & 18.39 \\
Mala & 1 & 2 & 3 & 3.45 \\
\hline Total & 46 & 41 & 87 & 100.00 \\
\hline
\end{tabular}

La atención médica se consideró buena en 80 casos (68.96\%), regular en 22 (25.29\%) y mala en 5 casos (5.75\%). Es decir hubo error en el diagnóstico de ingreso, en la evolución y/o en el manejo, que incidieron desfavorablemente en la recuperación de las enfermas en un $31.04 \%$ de los casos.

Tabla No. 29

ATENCION MEDICA

\begin{tabular}{lrrrr}
\hline Año & 1983 & 1984 & Total & Porcentaje \\
\hline Buena & 33 & 27 & 60 & 68.96 \\
Regular & 12 & 10 & 22 & 25.29 \\
Mala & 1 & 4 & 5 & 5.75 \\
\hline Total & 46 & 41 & 87 & 100.00 \\
\hline
\end{tabular}

\section{Atención Institucional:}

Se analiza en la tabla 30.

En el 28.73\% de las muertes maternas se consideró que existió algún grado de

Tabla No. 30

ATENCION INSTITUCIONAL

\begin{tabular}{lcrrr}
\hline Año & 1983 & $\mathbf{1 9 8 4}$ & Total & Porcentaje \\
\hline Buena & 35 & 27 & 62 & 71.27 \\
Regular & 11 & 13 & 24 & 27.59 \\
Mala & - & 1 & 1 & 1.14 \\
\hline Total & 46 & 41 & 87 & 100.00 \\
\hline
\end{tabular}


falla en la disponibilidad de recursos necesarios por parte del Hospital para la adecuada atención. Solo en un $1.14 \%$ la falla institucional influyó desfavorablemente en la evolución de las pacientes.

\section{Muerte Institucional:}

Se encontraron 26 casos de muerte no institucional (29.88\%) y 61 casos de muerte institucional (70.12\%). Estos datos se relacionan en la tabla 31 .

Tabla No. 31

\section{MUERTE INSTITUCIONAL}

\begin{tabular}{lcccr}
\hline Año & $\mathbf{1 9 8 3}$ & $\mathbf{1 9 8 4}$ & Total & Porcentaje \\
\hline Menos de 24 horas & 15 & 11 & 26 & 29.88 \\
Más de 24 horas & 31 & 30 & 61 & 70.12 \\
\hline Total & 46 & 41 & 87 & 100.00 \\
\hline
\end{tabular}

Para una mejor apreciación de los datos $y$ de la gravedad de la enfermedad de las pacientes, se encontró que 6 pacientes $(6.89 \%)$ fallecieron antes de 3 horas de permanencia en la Institución, 12 $(13.79 \%$ ) entre las 3 y las 12 horas y 8 $(9.20 \%)$ entre las 12 y 24 horas. La paciente que estuvo más tiempo hospitalizada, permaneció 38 días en la Institución.

\section{VIII - DISCUSION}

El análisis de la mortalidad materna ocurrida en el Hospital Materno Infantil, durante el lapso 1983-1984, está basado en la metodología seguida en los estudios realizados sobre este mismo aspecto en los años 1976-1980 y 1981-1982 $(3,15)$.

Hemos querido con el presente trabajo continuar la revisión sobre este campo, con el ánimo de mantener actualizada la situación sobre la atención materna, dispensada en el nivel universitario de la Regional No. 3 de Bogotá, en don- de se refleja a través de este indicador, la realidad que se vive en otras áreas de Bogotá y del país, que registran situaciones similares en razón al nivel de pacientes que se atienden $y$ los recursos disponibles en la solución de los problemas de salud.

Se incluye la revisión de variables no contenidas en otras investigaciones realizadas en la Institución, tales como datos al ingreso de las pacientes, datos y complicaciones del parto, complicaciones de las principales causas de muerte y un análisis de las anestesias recibidas por las pacientes fallecidas.

En el presente estudio se siguieron observando fallas en la historia clínica, que se habían señalado en estudios anteriores, tales como;

a. Elaboración incompleta de la información, con omisión de variables importantes que facilitarían posteriormente su análisis.

b. Error de diagnóstico al ingreso.

c. No se definen pautas de manejo y conducta a seguir con base en una sospecha clínica inicial.

d. En ocasiones no existe una epicrisis o cierre completo de la historia.

e. A veces no se valoran los datos contenidos en las hojas de remisión de otras instituciones.

f. Patología no justifica, en algunos casos, la omisión de la práctica de la autopsia, dificultando el análisis final de las muertes.

g. El hecho de haber 2 servicios de estadística independientes, uno en el Hospital San Juan de Dios y otro en el HMI, hace que ,no haya continuidad en los datos de la historia cl ínica y que en algunos 
casos éstas se pierdan o que se repitan estudios diagnósticos que ya se habían prácticado.

La situación planteada con las historias incompletas se subsanó en gran parte con la revisión exhaustiva de toda la documentación existente en cada caso, a partir del archivo de Anatomía Patológica, laboratorio clínico, !ibros de registro existentes en admisión, salas de parto, cirugía, servicios de sépticas y toxemias y cuidado intensivo, entre otros. De esta manera se obtuvo una información más completa, que junto con los análisis hechos en reuniones de mortalidad, permitió llegar a un juicio objetivo sobre cada muerte ocurrida en el HMI.

\section{Tasa de mortalidad:}

En los dos años revisados en el HMI (1983-1984), se presentaron ochenta $y$ siete (87) muertes maternas que cumplían los parámetros señalados por la FIGO, para ser consideradas como tales.

Durante el mismo lapso ocurrieron en la Institución veinte mil ochocientos noventa y un nacimientos (20.891) de niños vivos.

Relacionando estas dos cifras se obtuvo una tasa de mortalidad materna de $41.64 \times 10.000$ nacidos vivos.

La tasa anterior modifica la tendencia descendente de la mortalidad materna en el HMI, vista entre 1960 y 1980 y sobrepasa ampliamente la encontrada por Muñoz y Becerra en 1981-1982 de 32.64, período en el cual empezó a notarse un nuevo incremento de las cifras. La tasa encontrada en el presente estudio se acerca a la vista en la Institución, entre 1960 y 1965 , lo que nos indica la gravedad del fenómeno.

En la tabla 32 se señalan las tasas de mortalidad observadas en el $\mathrm{HMI}$ en los últimos 30 años. $(3,11,12,15,17,19$, $20,21)$.

Tabla No. 32

TASAS DE MORTALIDAD MATERNA EN EL HMI

\begin{tabular}{lll}
\hline Autor & Años & \multicolumn{1}{c}{ Tasa } \\
\hline Peral ta y colaboradores & $1954-57$ & $60.12 \times 10.000$ \\
López Ruiz & $1960-65$ & 45.4 \\
Rojas y colaboradores & $1966-70$ & 31.1 \\
Sánchez Torres & $1971-75$ & 30.47 \\
Muñoz y colaboradores & $1976-80$ & 25.04 \\
Muñoz y Becerra & $1981-82$ & 32.64 \\
Ñañez y Garcia & $1983-84$ & 41.64 \\
\hline
\end{tabular}

Al comparar la tasa de mortalidad anterior, con la encontrada en estudios realizados en otras instituciones universitarias y hospitales generales del país, se aprecia cómo nuestra institución tiene uno de los índices más altos de mortalidad. Ver tabla 33. $(4,9,10,23)$.

Tabla No. 33

\section{TASAS DE MORTALIDAD MATERNA EN HOSPITALES DE COLOMBIA}

\begin{tabular}{lcl}
\hline & Años & Tasa \\
\hline Hospital del Valle (Cali) & $1977-78$ & 42.9 \\
HMI (Bogotá) & $1983-84$ & 41.64 \\
$\begin{array}{l}\text { Hospital San Vicente de } \\
\text { Páúl (Medellín) }\end{array}$ & $1968-72$ & 27 \\
$\begin{array}{l}\text { Estudios hospitalarios } \\
\text { de mortalidad materna }\end{array}$ & $1979-80$ & 23.1 \\
\hline
\end{tabular}

Si cotejamos la cifra de mortalidad encontrada en esta revisión con la notificada por centros hospitalarios del área latinoamericana, comparables por muchos aspectos con nuestra Institución, encontramos aún más alta la tasa de mortalidad. Ver tabla $34(4,9,1,2$, $22,24)$.

La tasa encontrada en el HMI es extremadamente alta $y$ en crecimiento 
muy acelerado al relacionarla con instituciones hospitalarias de países desarrollados, en donde prácticamente se han controlado las causas determinantes de mortalidad. Ver tabla $35(2,5,14)$.

Tabla No. 34

TASAS DE MORTALIDAD MATERNA EN LATINOAMERICA

\begin{tabular}{llll}
\hline Hospital & Ciudad & Años & Tasa \\
\hline HMI & Bogotá & $1983-84$ & 41.64 \\
Hospital "Concepción Palacios" & Caracas & $1939-74$ & 15.9 \\
Hospital "20 de noviembre" & México & $1976-79$ & 14.60 \\
Hospital Universitario & Maracaibo & $1961-70$ & 14.20 \\
Hospital Central & Valencia & $1970-79$ & 10.8 \\
IMSS & México & $1976-$ & 10.75 \\
Hospital "Dr. José Gregorio & & & \\
Hernández" & Caracas & $1973-80$ & 5.18 \\
\hline
\end{tabular}

Tabia No. 35

\section{TASAS DE MORTALIDAD MATERNA EN PAISES DESARROLLADOS}

\begin{tabular}{llll}
\hline \multicolumn{1}{c}{ Hospital } & \multicolumn{1}{c}{ Ciudad } & Años & Tasa \\
\hline Pensilvania & Pensilvania & $1959-73$ & 3.4 \\
Hutzel & Detroit & $1976-80$ & 2.4 \\
Milwake & Wisconsin & 1977 & 1.86 \\
Landesfranenklinik & Wuppeutel & $1973-79$ & 1.6 \\
\hline
\end{tabular}

Más adelante analizamos los factores que pueden estar incidiendo en el ascenso vertiginoso en la tasa de mortalidad del HMI.

\section{Causas de mortalidad materna:}

El mayor número de muertes maternas se encontró en el grupo de causas directas, con un total de 69 casos, equivalente a 79.31 \% sobre la mortalidad global. Sigue en este orden el grupo de causas indirectas con 11 casos (12.64\%) y finalmente las causas de orden no obstétrico con 7 casos (8.05\%).

Al revisar las causas de muerte materna en el HMI, desde 1966 (tabla 36) se aprecia una disminución de las causas directas, con un incremento de las causas indirectas, especialmente en los últi$\operatorname{mos} 4$ años $(3,15,16,17,18)$.

Tabla No. 36

CAUSAS DE MORTALIDAD MATERNA

\begin{tabular}{llcl}
\hline \multicolumn{1}{c}{ Años } & \multicolumn{3}{c}{ PORCENTAJE } \\
\hline & Directa & Indirecta & No materna \\
\hline $1966-70$ & 88 & 5.5 & 6.5 \\
$1971-75$ & 87.26 & 5.26 & 7.48 \\
$1976-80$ & 87.8 & 9.9 & 2.3 \\
1981 & 86.11 & 13.88 & - \\
1982 & 90 & 3.33 & 6.66 \\
1983 & 78.26 & 13.04 & 8.70 \\
1984 & 80.49 & 12.19 & 7.32 \\
\hline
\end{tabular}

\section{Mortalidad materna de causa directa:}

Dentro de este grupo la infección ocupa el primer lugar $(62.31 \%)$, seguida por la toxemia (18.84\%), la hemorragia (10.14\%), el hígado graso (4.35\%), la embolia amniótica (2.89\%) y la enfermedad trofoblástica (1.45\%).

El ordenamiento de las 3 principales causas directas de muerte es igual al encontrado desde 1966 (Tabla 37), pero es la primera vez que se encuentran por-

Tabla No. 37

MORTALIDAD MATERNA DE CAUSA DIRECTA

\begin{tabular}{llll}
\hline \multicolumn{2}{l}{ Años } & \multicolumn{3}{c}{ PORCENTAJE } \\
\hline & Infección & Toxemia & Hemorragia \\
\hline $1966-70$ & 64 & 20.5 & 6.6 \\
$1971-73$ & 52 & 22.2 & 8 \\
$1971-75$ & 60.94 & 17.73 & 4.99 \\
$1976-80$ & 69.69 & 19.04 & 7.79 \\
$1981-82$ & 86.2 & 6.9 & 5.2 \\
$1983-84$ & 62.31 & 18.84 & 10.14 \\
\hline
\end{tabular}


centajes descendentes en la infección en los últimos 14 años. A su vez la hemorragia llega al nivel más al to encontrado en los últimos 6 estudios. La toxemia luego de un descenso marcado encontrado en el último estudio, vuelve a sus niveles previos $(3,15,16,17,18)$.

\section{a. Sepsis obstétrica:}

La infección en general mostró un porcentaje mucho mas bajo que en años anteriores. La infección por aborto continúa siendo la causa más importante de muerte materna en general (37.93\%) y se encontró el porcentaje más alto de éste (76.74\%) como causa de infección, de los últimos 5 estudios realizadós en el $\mathrm{HMI}$.

La infección en el puerperio y en el post-operatorio, mostró un descenso porcentual marcado con relación a los estudios mencionados. Ver tabla 38 (3, $12,19,20,21)$.

Tabla No. 38

MORTALIDAD MATERNA POR INFECCION

\begin{tabular}{|c|c|c|c|c|}
\hline \multirow[t]{2}{*}{ Años } & \multicolumn{3}{|c|}{ PORCENTAJE } & \multirow[b]{2}{*}{ Ectópico } \\
\hline & Aborto & Puerperio & Cesárea & \\
\hline $1966-70$ & 75 & 15 & 10 & \\
\hline $1971-73$ & 38.2 & 13.8 & 48 & \\
\hline $1976-80$ & 65.8 & 17.4 & 16.8 & \\
\hline $1981-82$ & 68 & 14 & 16 & 2 \\
\hline $1983-84$ & 76.74 & 11.63 & 9.30 & 2.23 \\
\hline
\end{tabular}

Al revisar las 9 muertes ocurridas en el puerperio a causa de la infección, se estableció que 5 de las pacientes fueron remitidas al $\mathrm{HMI}$ en estados sépticos avanzados después de parto (4) o cesárea (1) atendidos en hospitales de atención intermedia de Bogotá y de poblaciones vecinas. Las 4 pacientes restantes presentaron infecciones después de parto en 1 caso y cesárea en 3 casos, atendidos en el HMI.
Las cifras anteriores demuestran como el manejo es inadecuado y la remisión de pacientes sépticas no se hace en forma oportuna al nivel correspondiente.

A través de la revisión de las historias clínicas, se vio que la dependencia de la unidad de Cuidado Intensivo del Hospital San Juan de Dios y la limitación de camas de ese servicio, contribuyó en muchos casos a que las pacientes no fueran trasladadas o lo fueran tardiamente, permitiendo que las complicaciones de la infección siguieran su curso natural por falta de tratamiento intensivo, complementario del manejo quirúrgico del Servicio de Sépticas.

Lo anterior unido al grado de desnutrición, anemia, malas condiciones socioeconómicas y ambientales que tienen estas enfermas, hace que la sepsis tenga una evolución rápida y nefasta en la mayoría de ellas.

El problema del aborto es difícil de evaluar, ya que los estudios hospitalarios, es bien conocido, solo proporcionan información sobre una parte de los abortos que se suceden en de terminado lugar. Cuando el aborto cursa sin complicaciones, no se hospitaliza y muchos abortos aún no requieren atención médica, por lo cual es difícil conocer la magnitud del problema (18).

\section{b. Toxemias:}

En los últimos estudios de mortalidad realizados en el $\mathrm{HMI}$, se venía notando un descenso franco en el gruno de pacientes muertas por toxemia, que resaltaba especialmente en el período 1981. 1982. En el estudio actual la toxemia vuelve a sus niveles previos, demostrando un retroceso con relación a los 2 años del estudio anterior, $(3,15,16,17,18$, 25). 
A pesar de los esfuerzos realizados en el HMI, para mejorar el manejo de la toxemia, aún siguen llegando pacientes con embarazo a término y complicaciones irreversibles producidas por la entidad en referencia. Si tenemos en cuenta que el $81.60 \%$ del total de pacientes muertas, no tuvieron control prenatal, se puede deducir lo poco que se está haciendo para la prevención de esta enfermedad y se insiste, una vez más, en la importancia de mejorar la atención prenatal a nivel de los diferentes organismos de salud del pais, para detectar precozmente los síntomas y signos que obligarían a una pronta remisión de estas enfermas al nivel correspondiente $y$ una atención más oportuna.

\section{c. Hemorragia:}

La hemorragia luego de la disminución señalada por Muñoz y Becerra en 1981-1982, yolvió a presentar aumento con 7 casos $(10.14 \%)(3,15,19,20,21)$.

En su mayoría la hemorragia estuvo relacionada con el parto y se vieron nuevamente casos de desgarros en cesárea (2), atonía uterina (1), acretismo placentario (1), alumbramiento incompleto (1), abruptio placentae (1) y embarazo ectópico (1).

Con un mejor manejo de la conducción $\mathrm{y}$ atención del parto $\mathrm{y}$ conductas más activas en el manejo de las causas de hemorragia en el tercer trimestre del embarazo, se lograría reducir el número de muertes por ésta causa.

\section{Mortalidad Materna de causa indirecta:}

Dentro de este grupo la enfermedad vascular hipertensiva crónica (EVHC) está en primer lugar con 4 casos (36.36\%). Al compararla con las causas de mortalidad global ocupa el cuarto lugar con un $4.59 \%$ y al sumarla a la toxemia se ubican en el segundo puesto con un $19.54 \%$

Las cifras encontradas no muestran un cambio favorable en relación con los dos trabajos anteriores realizados en la Institución, encontrándose igual número de casos de enfermedad vascular hipertensiva crónica al período anterior, aunque el porcentaje disminuyó por la aparición de otras causas de muerte indirecta $(3,15)$.

$\mathrm{Si}$ analizamos en conjunto las causas indirectas de muerte materná en el presente estudio (enfermedad vascular hipertensiva crónica, enfermedad cardiorrespiratoria, tuberculosis, pielonefritis, glomerulonefritis, síndrome varicoso severo, leucemia, valvulopatía aórtica), podemos deducir que estas muertes se habrían evitado en al to porcentaje, si a estas mujeres se les hubiera advertido, sobre la gravedad de sus patologías previas y el riesgo inherente de una nueva gestación, que ha debido evitarse de manera temporal o definida.

\section{Muerte no materna:}

Dentro de las causas consideradas como no obstétricas en la mortalidad materna, encontramos dos accidentes anestésicos que fueron ampliamente analizados en la Institución y cuya ocurrencia pone de relieve la necesidad de registrar en trabajos posteriores otros eventos asociados a la anestesia, tales como el paro cardíaco, la muerte intraoperatoria y el accidente anestésico no mortal, que no se están cuantificando adecuadamente en la Institución.

Entre los demás casos encontramos 2 tumorales, uno de ellos un carcinoma de cérvix en estado terminal, en que la paciente fallece por infección facilitada por las pésimas condiciones generales en que se encontraba desde su ingreso; un caso de un osteosarcoma, en que la pa- 
ciente muere por complicaciones de la neoplasia, cursando además el segundo trimestre de la gestación.

Se encontró un caso de muerte por estatus convulsivo en una paciente que cursaba el primer trimestre de embarazo.

En los otros dos casos se confundió la ruptura de un aneurisma y un caso de meningitis, con las complicaciones neurológicas de la toxemia. Se pone de presente una vez mas, que no se debe olvidar que la mujer en estado grávido-puerperal puede presentar cualquier patología médico-quirúrgica, que si bien en ocasiones se enmascara por el embarazo, obliga al clínico a agudizar sus sentidos, con él ánimo de hacer un diagnóstico oportuno y un manejo más adecuado $y$ en equipo interdisciplinario para tratar de evitar estas muertes.

\section{Causa de mortaiidad materna en rela- ción a: edad, número de gestaciones y estadío del embarazo:}

La mayor mortalidad en relación con edad materna, se presentó en el grupo de 20 a 29 años $(52.87 \%$ ) a causa de la infección derivada principalmente del aborto provocado, la toxemia, la enfermedad vascular hipertensiva crónica y la hemorragia. Sigue en orden de frecuencia el grupo de 30 a 34 años, por las mismas causas.

Al relacionar las variables número de gestaciones y causa de muerte, se aprecia como las pacientes con 3 o más hijos, junto con aquellas que cursaban su primer embarazo tienen los índices más altos $(52.88 \%$ y $24.14 \%$, respectivamente).

La causa más frecuente en las multíparas fue la infección, a expensas principalmente del aborto provocado. En el grupo de primigestantes fue más frecuente la toxemia pero seguida de cerca por la infección.
Los estadíos del embarazo en donde ocurrió mayor mortalidad fueron primer trimestre de la gestación (40.23\%), puerperio (25.29\%) y tercer trimestre (25.29\%). En el primer grupo la causa de muerte fue en su mayoría la infección post-aborto provocado. En el segundo grupo la infección puerperal y en el último grupo las complicaciones de la toxemia y la enfermedad vascular hipertensiva crónica.

Es preocupante el alto número de muertes por infección, principalmente originadas por el aborto provocado, que es utilizado como "método de planificación" por mujeres en cualquier edad reproductiva y que dado en las pésimas condiciones en que se practica, hace que nuestras mujeres de estratos socioeconómicos bajos, paguen con su vida y la desintegración del núcleo familiar, la interrupción del embarazo.

Llama la atención el elevado número de mujeres que mueren en el puerperio a consecuencia principalmente de infección y hemorragia, derivados de un mal manejo en la atención del parto o cesárea; ambas situaciones atribuibles en parte a las Instituciones Hospitalarias y a su cuerpo médico que interviene en el manejo de estas pacientes.

\section{Anatomía patológica:}

La au topsia sola o complementada con estudio de pieza quirúrgica se hizo en 55 casos (63.22\%). La autopsia se realizó como procedimiento único en 47 pacientes $(54.02 \%)$.

Los hallazgos quirúrgicos encontrados durante intervenciones practicadas antes de la muerte a un grupo de 8 pacientes (9.20\%) y su correspondiente estudio his tológico de los especímenes extirpados, permitió una amplia confirmación clinico-patológica del diagnóstico. Este hecho fue tenido en cuenta por los patólogos, 
quienes no justificaron practicar estudio post-morten en estos casos. A otras 8 pacientes $(9.20 \%)$ se les practicó autopsia a pesar de tener estudio de pieza quirúrgica, para complementar así el análisis de cada caso.

No tuvieron estudio anatomo-patológico 24 pacientes $(27.58 \%)$, por levantamiento médico legal en 16 casos (18.39\%), por oposición familiar en 3 casos (3.45\%) y no se pudo conocer la razón por la cual no se practicó el estudio en 5 ca$\operatorname{sos}(5.74 \%)$.

\section{Mortalidad materna en relación con remisión de pacientes:}

Sobre el total de las 87 pacientes fallecidas, solamente $6(6.89 \%)$ consultaron directamente a la Institución, en 22 no hubo dato de remisión (25.28\%) y se consideraron como pacientes nuevas en la Institución. Las otras 59 enfermas (67.81\%) Ilegaron remitidas en su mayoría de hospitales regionales (19.54\%), hospitales locales $(9.19 \%)$, hospitales universitarios $(6.89 \%)$, clínicas $(4.59 \%)$ y médicos particulares $(1.14 \%)$. Estos datos ponen de presente, una vez más como el HMI es un hospital de referencia de cualquier zona de la ciudad y poblaciones vecinas.

Desaforturadamente en el Distrito Especial de Bogotá la regionalización en la atención de salud y la remisión de pacientes no opera en la forma establecida por el Sistema Nacional de Salud y menos aún teniendo en cuenta la sectorización en que se encuentra dividida la ciudad, en base a los diferentes niveles de atención existentes.

En la tabla 39 se observa cómo los hospitales regionales han incrementado 4 veces el número de remisiones y los hospitales universitarios las han duplicado. No se indica el número de pacientes que procedían de su área de influencia por lo cual las cifras reales son mucho más al tas $(3,15)$.

Tabla No. 39

LUGAR DE REMISION

\begin{tabular}{lrcr}
\hline Años & $\mathbf{1 9 7 6 - 8 0}$ & $\mathbf{1 9 8 1 - 8 2}$ & $\mathbf{1 9 8 3 - 8 4}$ \\
\hline Directamente HMI & 60.83 & 50.00 & 6.89 \\
Centro de Salud & 10.27 & 9.09 & 19.54 \\
Hospital Regional & 6.08 & 22.72 & 26.43 \\
Empírico & 5.32 & - & \multicolumn{1}{l}{-} \\
Hospital local & 4.94 & 13.63 & 9.19 \\
Médico particular & 4.56 & - & 1.14 \\
Hospital Universitario & 3.80 & 4.54 & 6.89 \\
Clínica & 2.66 & - & 4.59 \\
Sin Dato & 1.53 & - & 25.28 \\
\hline
\end{tabular}

La falla anotada en la aplicación de la regionalización hace que no se preste en muchos casos la atención de alto riesgo obstétrico en forma oportuna a pacientes de escasos recursos y que al ser remitidas al HMI la Institución soporte una carga asistencial mayor a sus propias capacidades y recursos disponibles. Ademas, al recibir enfermas remitidas con complicaciones irreversibles, eleva su tasa de mortalidad, sin que ello refleje de manera objetiva la calidad de la atención ofrecida por la Institución.

\section{Mortalidad materna en relación con número de nacimientos $y$ número de muertes maternas por año:}

En la tabla 40 se observa como el HMI en los últimos 4 años disminuyó a la mitad el número de nacimientos de niños vivos por año, al tiempo que el número de muertes maternas por año sufrió una ligera variación aunque con tendencia al descenso. Esto lleva a que al disminuir el denominador, aumenten las tasas de mortalidad y se espera que continúen aumentando si el número de muertes sigue aumentando, con el consiguiente aumento en el numerador, $(3,15,19$, $20,21)$. 
Tabla No. 40

NACIMIENTOS Y MUERTES MATERNAS POR AÑO EN EL HMI

\begin{tabular}{cccl}
\hline Años & Nacimientos & Muertes maternas & Tasa \\
\hline 1971 & 23.402 & 64 & 27.3 \\
1972 & 24.467 & 61 & 24.9 \\
1973 & 21.061 & 84 & 39.8 \\
1976 & 22.275 & 59 & 26.57 \\
1977 & 20.769 & 60 & 28.98 \\
1978 & 21.421 & 53 & 24.76 \\
1979 & 20.869 & 40 & 19.23 \\
1980 & 19.761 & 51 & 26.02 \\
1981 & 9.381 & 36 & 38.37 \\
1982 & 11.143 & 30 & 26.92 \\
1983 & 10.796 & 46 & 42.60 \\
1984 & 10.095 & 41 & 40.61 \\
\hline
\end{tabular}

10. Atención institucional y méciica:

No es fácil determinar con precisión la existencia de responsabilidad en la atención de una enferma por parte de una institución o del personal médico que tuvo a su cargo su manejo.

Es muy difícil evaluar la atención medica e institucional, ya que la veracidad de los hechos en el manejo de una paciente depende en buena parte de la calidad de la historia clínica y de muchas otras circunstancias que algunas veces no se conocen $y$ que influyen en la evolución de cada caso.

También hay que tener en cuenta factores de la paciente, tales como culturales, sociales, económicos y religiosos que pueden incidir en forma desfavorable en la progresión de su enfermedad.

Dejando de lado las consideraciones anteriores, es útil para el Hospital y el Departamento de Ginecología y Obstetricia, establecer mediante este indicador de atención institucional y médica, una vigilancia permanente, cuyo objetivo principal debe ser el despertar en todos los estamentos de la Institución el sentido de responsabilidad que a cada uno de ellos compete.

A través de la revisión de cada historia clínica, se estableció cómo la falta de recursos institucionales necesarios en la atención de las pacientes, contribuyó en un $28.73 \%$ de manera desfavorable en la evolución de las enfermas. De igual manera se encontró, en relación con la atención médica que el diagnóstico equivocado en algunos casos, seguimien to inadecuado en otros o la demora en la aplicación del tratamiento médico o quirúrgico, favoreció el empeoramiento del estado general de las pacientes en un $31.04 \%$.

En las tablas 41 y 42 , se señala cómo gn relación con los dos últimos trabajos de mortalidad realizados en el $\mathrm{HMI}$, las fallas institucionales tienden a disminuir, al igual que las fallas médicas, pero sin tener cifras que se puedan considerar adecuadas en un hospital universitario responsable de la atención de alto riesgo obstétrico $(3,15)$.

Tabla No. 41

ATENCION INSTITUCIONAL

\begin{tabular}{lccr}
\hline Año & $1976-80$ & $1981-82$ & $1983-84$ \\
\hline Buena & 67.68 & 69.7 & 71.27 \\
Regular & 23.19 & 19.6 & 27.59 \\
Mala & 9.12 & 10.7 & 1.14 \\
\hline
\end{tabular}

Tabla No. 42

ATENCION MEDICA

\begin{tabular}{lccr}
\hline Año & $1976-80$ & $1981-82$ & $1983-\mathbf{8 4}$ \\
\hline Buena & 49.8 & 68.2 & 68.96 \\
Regular & 31.5 & 18.2 & 25.29 \\
Mala & 18.6 & 13.6 & 5.75 \\
\hline
\end{tabular}




\section{Tasa Depurada de Mortaliciad materna en el HMI:}

Creimos importante tratar de establecer el número de enfermas, cuya causa de muerte sea atribuible directamente al Hospital, en razón al manejo y cuidados dispensados dentro de la Institución. De acuerdo al comentario hecho anteriormente en relación con las pacientes remitidas al HMI, así como las que ingresaron directamente a él, se estableció que en 28 pacientes $(32.19 \%)$ sobre el gran total de las 87 fallecidas, ia complicación obstétrica que las lievó a la muerte se debió al manejo dado en el Hospital. Al correlacionar el total de muertes atribuibles al $\mathrm{HMI}$ con el número de nacidos vivos en los dos años analizados (20.891), tendríamos una tasa de mortalidad depurada de 13.40 por 10.000 nacidos vivos, cifra esta que sí refleja la calidad de la atención dispensada por el nivel universitario de la Regional 3.

\section{IX - CONCLUSIONES}

1. La tasa de mortalidad materna en el Hospital Materno Infantil de Bogotá durante los años 1983-1984 fue de 41.64 por 10.000 nacidos vivos.

2. Durante los dos años analizados (1983-1984) se observó un marcado aumento en la tasa de mortalidad de la Institución.

3. La mortalidad más alta se encontró en el grupo de causas directas (79.31\%), seguidas de las causas indirectas (12.64\%) y las no obstétricas (8.05\%).

4. Entre las causas directas la infección ocupa el primer lugar (62.31\%) seguida por la toxemia (18.84\%), la hemorragia (10.14\%), el hígado graso (4.35\%), la embolia amniótica (2.89\%) y la enfermedad trofoblástica (1.45\%).

5. El aborto provocado es la principal causa de sepsis obstétrica (76.74\%).
6. Es alto el porcentaje de infección puerperal (20.94\%) a partir de parto o cesárea.

7. Los estados sépticos más frecuentes fueron: la miometritis (55.81\%), la peritonitis (53.48\%) y el shok séptico (51.16).

8. Las complicaciones más frecuentes de la infección fueron: el síndrome de dificultad respiratoria del adulto (32.55\%), la insuficiencia renal aguda $(32.55 \% i$, el tromboembolismo pulmonar $(27.90 \%$ ), la trombosis de infundíbulopélvico (23.25\%) y el edema pulmonar (18.60\%). Estas entidades requieren para su manejo adecuado una Unidad de Cuidado Intensivo, de la cual actualmente no dispone la Institución.

9. La toxemia ocupó el segundo lugar como causa de muerte en general $(14.94 \%)$, y mostró un incremento marcado con relación a los dos años anteriores en que se había visto descenso ostensible.

10. Las complicaciones más frecuentes de la toxemia fueron: la insuficiencia renal aguda (76.92\%), el edema pulmonar $(69.23 \%)$, el accidente cerebro-vascular (69.23\%), edema cerebral (53.85\%) y la hemorragia subcapsularhepática (46.15\%).

11. La hemorragia luego de un descenso observado en los dos años anteriores, volvió a mostrar incremento por patológía asociada al parto principalmente.

12. La mortalidad materna de causa indirecta está dada principalmente por la enfermedad vascular hipertensiva crónica, que ocupa junto con la toxemia el segundo lugar dentro de las causas globales.

13. La mortalidad materna de causa indirecta se considera evitable en la mayoi ía de los casos. 
14. El accidente anestésico es la causa principal de muerte no materna (28.57\%).

15. Eventos asociados a la anestesia tales como el paro cardíaco, la muerte in. traoperatoria, el accidente anestésico no mortal y el accidente anestésico mortal, se presentaron en el $25.28 \%$ de las pacientes fallecidas en los dos años del estudio.

16. En los grupos de 20 a 24 años (28.73\%) y de 25 a 29 años (22.99\%), se presentó la mayor mortalidad a causa del aborto provocado.

17. En las pacientes multiparas (48.28\%) se encontró el índice más al to de mortalidad a expensas del aborto provocado.

18. En pacientes primigestantes (24.14\%) se encontró el índice más alto de mortalidad a expensas de la toxemia.

19. La muerte ocurrió con mayor frecuencia en el primer trimestre de gestación (40.23\%), puerperio (25.29\%) y tercer trimestre (25.29\%).

20. No tuvieron control prenatal el $81.60 \%$ de las pacientes fallecidas en los dos años.

21. De las pacientes que tuvieron el parto $(60.91 \%)$ tuvieron una o más complicaciones relacionadas con el mismo el $69.81 \%$ y se presentó mortalidad perinatal en el $33.96 \%$

22. El $67.81 \%$ de las pacientes fallecidas fueron remitidas de otras instituciones al HMI, con complicaciones severas establecidas.

23. El aumento en el número de remisiones de pacientes complicadas y la disminución en el número de nacimientos, son responsables en buena parte del ascenso vertiginoso de la mortalidad observada en el $\mathrm{HMI}$ en los últimos 2 años.
24. La atención institucional y médica contribuyó desfavorablemente en el resultado final en un $1.14 \%$ y $5.75 \%$, respectivamente.

25. Se establece una tasa depurada de mortalidad materna en el $\mathrm{HMI}$ de 13.40, por 10.000 nacidos vivos.

\section{$X$ - RECOMENDACIONES}

El análisis de los resultados obtenidos en el presente estudio muestra una vez más cómo la mortalidad en nuestro país continúa siendo un problema de salud pública de gran magnitud, en donde a pesar de existir políticas de atención materno-infantil muy definidas por parte del Ministerio y Seccional de Salud y de los Departamentos Universitarios de Obstetricia y Ginecología, éstas no se reflejan en beneficios concretos sobre el grupo etário hacia el cual están orientados.

Lo anterior se demuestra fácilmente al ver cómo la tasa de mortalidad registrada en las instituciones como la nuestra, continúa aumentando en forma alarmante y se mantienen sin cambio los factores que están incidiendo directamente en el fallecimiento de la mujer gestante.

Sería importante buscar mecanismos que permitan integrar a través de la sección materno-infantil del Ministerio de Salud, el recurso humano de los diferentes organismos destinados a la atención materna, quienes en base a politicas definidas y programación académica elaboren $y$ coordinen planes conjuntos para desarrollar a través de recursos físicos y humanos disponibles en cada Regional. Esto conducirá a una mejor docencia y necesariamente una mejor atención a la comunidad.

Si se logra establecer la integración docente-asistencial, será posible integrar. y capacitar a todo el personal participan- 
te del equipo en los diferentes programas dirigidos a la comunidad, tales como control prenatal, planificación familiar, atención obstétrica y post-parto, consejería en reproducción humana y educación en salud, en los cuales actualmente hay graves fallas que no se están corrigiendo, dado que las cifras de mortalidad siguen aumentando.

Una vez lograda la unificación de los criterios y las conductas que se deben seguir en cada paciente frente a una entidad definida, cada Institución debe asumir la atención de las pacientes que le corresponden según lo establezca la clasificación de riesgo y la sectorización señalada por el Sistema Nacional de Salud.

En relación con el recurso Institucional disponible en cada nivel de atención, se hace necesario establecer o mejorar servicios que permitan prestar una atención integral $y$ oportuna en hospitales de atención secundaria y terciaria. Entre estos servicios vale la pena mencionar el de sépticas y toxemias. Sólo así podemos lograr que pacientes vistas en instituciones diferentes el HMI, puedan ser manejadas en la mayoría de los casos en estos organismos en forma oportuna y adecuada, evitando su remisión tardía cuando ya las secuelas establecidas son irreversibles.

El aumerito del aborto como causa de mortalidad es alarmante y sabemos que la solución del problema es muy compleja por existir dentro de él factores de diferente índole que no están a la mano del médico para resolverlos. Aparte de la educación integral en salud que ya hemos mencionado $y$ que comprende necesaria- mente la educación sexual y planificación familiar, es importante que el $\mathrm{Mi}$ nisterio y los Servicios de salud ejerzan un control permanente sobre el ejercicio ilegal de la profesión practicado por personal médico y empírico, quienes son responsables de las complicaciones derivadas del aborto.

Las muertes de causa indirecta, se pueden evitar a través de la motivación directa hecha en pacientes detectadas como de altísimo riesgo para una nueva gestación y lograr la aceptación de evitarla en forma temporal o permanente.

Se sugiere la creación en el HMl de una Unidad de Cuidado Intensivo, que complemente el manejo de pacientes en estado crítico especialmente de los servicios de sépticas y toxemias.

En relación con los estudios de mortalidad materna que se desarrollarán en el futuro en el HMI, es necesario recomendar la aceptación de un formulario que contenga las variables nuevas analizadas en el presente estudio y tan importantes como datos al ingreso, complicaciones de las principales causas de muerte y complicaciones relacionadas con el parto y con la anestesia.

El comité permanente de mortalidad debe solicitar, una vez ocurrida la muerte de una enferma, el diligenciamiento del formulario por parte del médico que cierra la historia y su envío inmediato para un análisis más exacto que facilite los estudios que sobre mortalidad materna se hagan posteriormente en la Institución. 


\section{ESTUDIO DE MORTALIDAD MATERNA EN EL IMI}

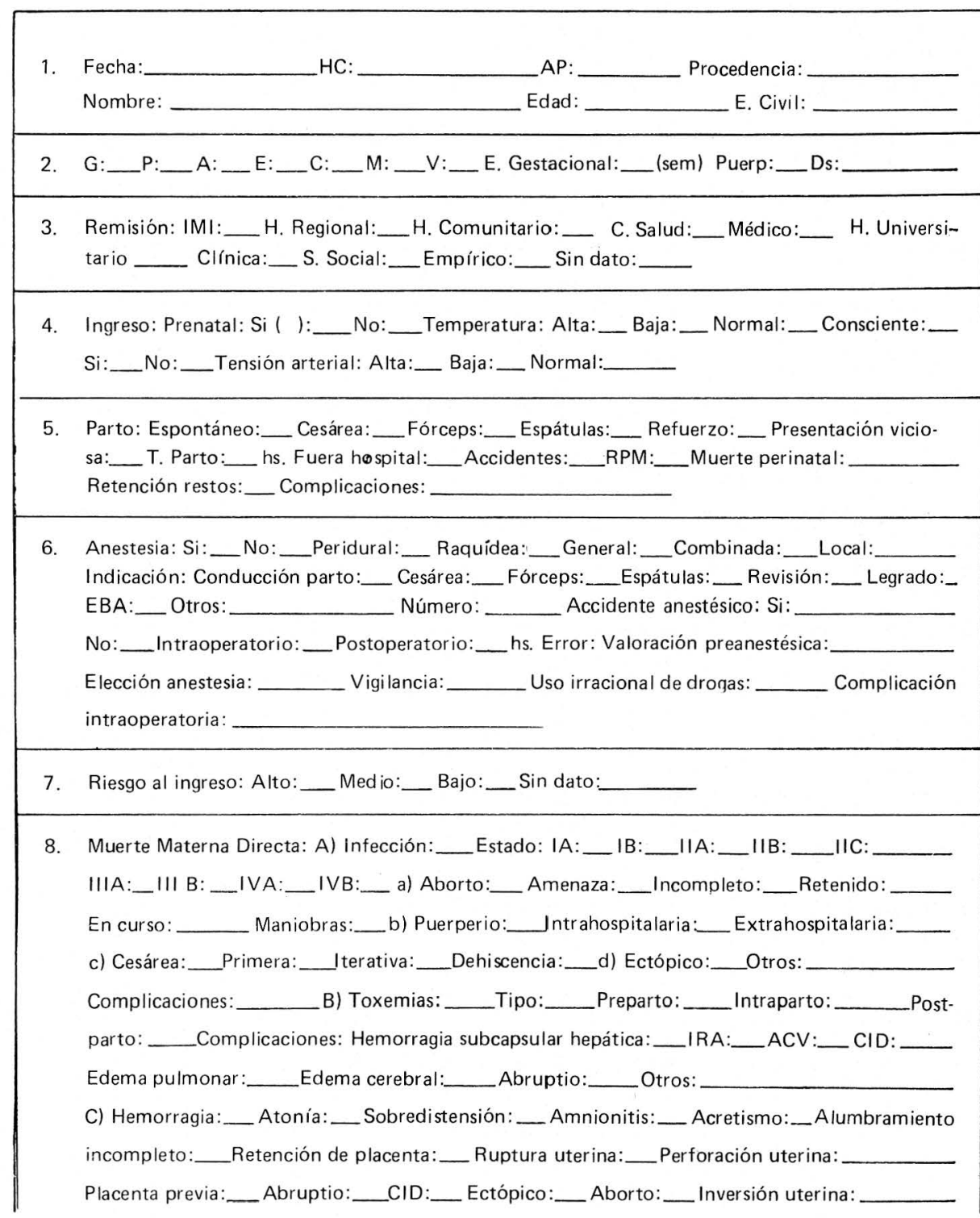




\section{(Continuación)}

Otros:

D) Embolia Amniótica:

E) Hígado graso:

Coriocarcinoma: Otros:

9. Muerte Materna Indirecta: Cardiorrespiratorias: _EVHC:

Renales:__IRA:__ Glomerulonefritis:__Otros:

Parasitarias:___ Virales:___ Colágeno:___ TEP: reumática:__ Púrpura trombocitopénica:__Otros:

TBC:__ Otros: Tumorales:

10. Muerte no materna: Intoxicación exógena: Anafilaxia: Suicidio: Coma de origen desconocido: Trauma:__ACV:__Cáncer:__Litiasis:__Aneurisma:__Paludismo: Edema pulmonar:__Accidente anestésico:__Neumonía:__Apendicitis:__ Gangrena: Infarto: Otros:

11. Diagnóstico Clínico: Acertado: Errado al principio: Errado al final:

12. Estancia:___ días. En un servicio:__En más de un servicio:

13. Historia: Buena: Regular: Mala: Comentarios:

14. Anatomía Patológica: Autopsia:___ Pieza quirúrgica:__ Medicina legal:___ Citología: Biopsia:___ Sin dato:____tros:

No autopsia por: Falta patólogo:__Neglicencia patólogo:__ M. legal:__ Oposición familiar: Pieza quirúrgica:__Deficiencias locativas: Exceso de trabajo: Comentarios:

15. Atención Médica: Buena:__Regular:__ Mala:__Nula:__Comentarios:

16. Muerte Institucional: 24 horas: 24 horas:

Atención Institucional: Buena:___ Regular:__ Mala:___Comentarios:

17. Diagnóstico final: Clínico:

Detinitivo:

18. Servicio: Cama: Otros datos:

19. Muerte: Evitable: Inevitable: Comentarios:

20. Fecha Revisión: Caso concluido: Revisión completa:

Comentarios: 


\section{XI-BIBLIOGRAFIA}

1. AUQUERO y Cols. Mortalidad materna en el Hospital Concepción Palacios de Caracas (1964-1972). Obstetricia y Ginecología Mexicana, 35-509, 1984.

2. ALVAREZ BRAVO. Mortalidad materna: Los estudios de mortalidad materna en el mundo actual. Gaceta Médica Mexicana. 104: 25-30, 1972.

3. BECERRA CORREDOR. Mortalidad Materna en el Hospital Materno Infantil de Bogotá (HMI): 1981-1982.

4. DIAZ LLANOS. Mortalidad Materna en el Hospital Universitario del Valle. Acta Médica del Valle. Vol. 19, No. 3,4-112, $118,1979$.

5. DIETEL H. y KEDING G. "Mutterssier -blichkeit- auch heute noch ein Problem?". Geburtsh. U. Frauenheilk, 36: $1,1976$.

6. GARCIA y Cols. Mortalidad Materna en el Hospital Provincial de Maternidad de Buenos Aires. Obstetricia y Ginecología Latinoamericana. 29: 478, 1971.

7. GUHA RAY. Maternal mortality in Urban Hospital Maggicc Women's Hospital. Obstetric and Gynecology 17: 430, 1976.

8. International Federation of Gynecology and Obstetric. Report on the activity of the Comitee on the Maternal Mortality. Journal International Federation Obstetric and Gynecology. 5: 302, 1967.

9. JUBIZ y Cols. Muertes Maternas en el Hospital San Vicente de Paul. Revista Colombiana de Obstetricia y Ginecología. Vol. XXIX No. 4, 178-82, 1978.

10. JUBIZ A. Y SANCHEZ C. Mortalidad Materna comparativa entre dos períodos 1963-67, 1968-72. Revista Colombiana de Obstetricia y Ginecología. Vol. XXVII, No. 3, 129-36, 1976.

11. LOPEZ RUIZ. Morbilidad y mortalidad en el Instituto Materno Infantil. Revista Colombiana de Obstetricia y Ginecología. Vol. XIX, No. 4, julio-agosto de 1968.

12. LOZANO C. y Cols. Morbimortalidad, clasificación y tratamiento del aborto séptico. Revista Colombiana de Obstetricia y Ginecología. Vol. XXXII, No. 2, marzo-abril de 1981.

13. LOZADA y Cols. Mortalidad Materna en el Hospital de Arequipa. Revista Peruana. Obstetricia y Ginecología, 15: 69, 1969.

14. MARMOL JG. History of the maternal mortality comitee in the USA.

15. MUÑOZ LA y ÑAÑEZ $H$. Mortalidad Materna en el Hospital Materno Infantil de Bogotá (HMI). Revista Facultad de Medicina, Universidad Nacional, 1985.

16. O'CONNORS TIMOTHY. Mortalidad Perinatal evitable. Urgencias Obstétricas. Salvat, 1982.

17. PERALTA Y Cols. Estudio de Mortalidad en el HMI. Revista Colombiana de Obstetricia y Ginecología. Vol. IX, enerofebrero de 1968.

18. RIZO A. y PRADA E. Desconocimiento sobre la magnitud del aborto en Colombia. Revista Colombiana de Obstetricia y Ginecología. Vol. XXXIII, No. 2, 94102, marzo-abril de 1982.

19. ROJAS LAURA y Cols. Mortalidad Materna en el HMI de Bogotá. Revista Colombiana de Obstetricia y Ginecología. Vol. XXV, No. 2, 128-49, marzo-abril de 1974.

20. SANCHEZ TORRES. Mortalidad Materna en el IMI de Bogotá. 1971-1973. Revista Colombiana de Obstetricia y Ginecología. Vol. XXV, No. 6, 395-401, 1974. 
21. SANCHEZ TORRES. Mortalidad Materna en el IMI. 1971-1975. Revista Colombiana de Obstetricia y Ginecología. Vol. XXVIII, No. 6, 217-228, 1977.

22. SUKERMAN y Cols. Mortalidad Materna por sepsis 1970-1979, Hospital Central de Valencia, Venezuela. Revista de Obstetricia y Ginecología de Venezuela. Vol. 41, No. 2, 95-102, 1981

23. URIZA y Cols. Estudio Hospitalario de mortalidad materna. Revista Colombiana de Obstetricia y Ginecología. Vol.
XXXIII, No. 5, 325-36. Septiembreoctubre de 1982.

24. UZCATEGUI O. y SILVA D. Mortalidad Materna en el Hospital "Dr. José Gregorio Hernández" de Caracas. Revista de Obstetricia y Ginecología de Venezuela, Vol. 41, No. 4, 233-36, 1981.

25. VELASCO A. y BARRIGA H. Mortalidad Materna y perinatal en los estados hipertensivos del embarazo. Revista Colombiana de Obstetricia y Ginecología. Vol. $X X X V$, No. 1, 13-35, enero-febrero de 1984. 